\title{
Operator-Schmidt decomposition of the quantum Fourier transform on $\mathbb{C}^{N_{1}} \otimes \mathbb{C}^{N_{2}}$
}

\author{
Jon Tyson* \\ Jefferson Lab, Harvard University, Cambridge, MA $02138 \mathrm{USA}^{\dagger}$
}

April 25, 2003

\begin{abstract}
Operator-Schmidt decompositions of the quantum Fourier transform on $\mathbb{C}^{N_{1}} \otimes \mathbb{C}^{N_{2}}$ are computed for all $N_{1}, N_{2} \geq 2$. The decomposition is shown to be completely degenerate when $N_{1}$ is a factor of $N_{2}$ and when $N_{1}>N_{2}$. The first known special case, $N_{1}=N_{2}=2^{n}$, was computed by Nielsen in his study of the communication cost of computing the quantum Fourier transform of a collection of qubits equally distributed between two parties. [M. A. Nielsen, PhD Thesis, University of New Mexico (1998), Chapter 6, arXiv:quant-ph/0011036] More generally, the special case $N_{1}=2^{n_{1}} \leq 2^{n_{2}}=N_{2}$ was computed by Nielsen et. al. in their study of strength measures of quantum operations. [M. A. Nielsen et. al, (accepted for publication in Phys. Rev. A); arXiv:quant-ph/0208077] Given the Schmidt decompositions presented here, it follows that in all cases the bipartite communication cost of exact computation of the quantum Fourier transform is maximal.
\end{abstract}

PACS numbers: 03.67.-a

*Email: jonetyson@post.harvard.edu

${ }^{\dagger}$ current address 


\section{Introduction}

Operator-Schmidt decompositions are useful for quantifying the non-local nature of operators on finite-dimensional bipartite Hilbert spaces. The first special cases of Schmidt decompositions of the quantum Fourier transform were computed by Nielsen [1] to illustrate his study of coherent quantum communication complexity. He considered the following problem:

Suppose Alice is in possession of $m$ qubits, Bob is in possession of $n$ qubits, and they wish to perform some general unitary operation $U$ which acts on their $m+n$ qubits. How many qubits must be communicated between Alice and Bob for them to achieve this goal?

Nielsen proved that the number $Q_{0}(U)$ of such qubits was bounded by

$$
1 / 2 \times K_{\text {Har }}(U) \leq Q_{0}(U) \leq 2 \min (n, m),
$$

where the Hartley strength $K_{\mathrm{Har}}$ satisfies

$$
K_{\text {Har }}(U) \equiv \log _{2}(\operatorname{Sch}(U)),
$$

where $\operatorname{Sch}(U)$, defined in Definition 4 below, is the number of nonzero Schmidt coefficients of $U$. The upper bound of (1) is trivial, for Alice could simply send her qubits to Bob and let him send them back after performing $U$, or vice-versa. To illustrate his theorem, Nielsen considered the quantum Fourier transform $\mathcal{F}_{2^{n} \times 2^{n}}$ on $n+n$ qubits. He showed that $K_{\text {Har }}\left(\mathcal{F}_{2^{n} \times 2^{n}}\right)=2 n$, yielding $n \leq Q_{0}\left(\mathcal{F}_{2^{n} \times 2^{n}}\right) \leq 2 n$. Subsequent work by Nielsen [2] improved the general lower bound of (11) by a factor of two, ${ }^{1}$ in particular implying that

$$
Q_{0}\left(\mathcal{F}_{2^{n} \times 2^{n}}\right)=2 n .
$$

In a later paper 3, Nielsen and collaborators further employ operator Schmidt decompositions in the quantitative study of strength measures of the nonlocal action of unitary operators. ${ }^{2}$ Besides revisiting the Hartley strength, among the several strength measures considered is the Schmidt strength,

$$
K_{\mathrm{Sch}}(U)=H\left(\left\{\frac{\lambda_{k}^{2}}{\operatorname{dim}(\mathcal{H} \otimes \mathcal{K})}\right\}\right)
$$

where $U$ is a unitary operator on $\mathcal{H} \otimes \mathcal{K},\left\{\lambda_{k}\right\}$ are its Schmidt coefficients, and $H$ is the Shannon entropy. They give a Schmidt decomposition of $\mathcal{F}_{2^{m} \times 2^{n}}$ on $m+n$ qubits for the case $m \leq n$ and conjecture that $\operatorname{Sch}\left(\mathcal{F}_{2^{m} \times 2^{n}}\right)=2^{2 n}$ for $m>n$.

\footnotetext{
${ }^{1}$ See also footnote 6 for a brief outline of an alternative proof. We remark that Nielsen considers qubits for convenience only. In particular, let $V$ be a unitary on $\mathbb{C}^{N_{1}} \otimes C^{N_{2}}$, where $N_{1}$ and $N_{2}$ are the respective dimensions of Alice and Bob's quantum states, with no requirement that $N_{1}$ and $N_{2}$ be a powers of two. Then any quantum computation of $V$ employing some combination of qudit communication and ancillae, possibly of varying dimension, satisfies the following bound: $\sum_{d=2}^{\infty} N_{d} \log _{2}(d) \geq K_{\text {har }}(V)$, where $N_{d}$ is the number of qudits of dimension $d$ communicated between Alice and Bob. It is assumed that at the end of the computation that Alice and Bob retain posession of their (now altered) data qudits, although the bound holds whether or not a given net transfer of the (restored) ancillae is allowed.

${ }^{2}$ They also consider more general quantum operations than unitaries.
} 


\subsection{Results}

Schmidt decompositions of the quantum Fourier transform $\mathcal{F}_{N_{1} \times N_{2}}: \mathbb{C}^{N_{1}} \otimes$ $\mathbb{C}^{N_{2}} \rightarrow \mathbb{C}^{N_{1}} \otimes \mathbb{C}^{N_{2}}$ are given for all $N_{1}, N_{2}>1$, with no requirement that either $N_{1}$ or $N_{2}$ be a power of two. As a special case, the conjecture of Nielsen and collaborators is affirmed. In all cases, the results of Nielsen imply that the bipartite communication cost of exact computation of the quantum Fourier transform is maximal. Once stated, the decomposition is easily verified; a short derivation is given in the Appendix.

\subsection{Definitions and Notation}

Definition 1 Let $N, N_{1}, N_{2}$ be integers greater than one satisfying $N=N_{1} N_{2}$. The quantum Fourier transformation ${ }^{3} \mathcal{F}_{N}: \mathbb{C}^{N} \rightarrow \mathbb{C}^{N}$ is the unitary operator satisfying

$$
\mathcal{F}_{N}|s\rangle_{N}=\frac{1}{\sqrt{N}} \sum_{t=0}^{N-1} \exp \left(\frac{2 \pi i}{N} t s\right)|t\rangle_{N}, s \in\{0, \ldots, N-1\},
$$

where each $|s\rangle_{N}$ is a standard basis element. The quantum Fourier transformation $\mathcal{F}_{N_{1} \times N_{2}}$ on $\mathbb{C}^{N_{1}} \otimes \mathbb{C}^{N_{2}}$ is obtained by identifying $\mathbb{C}^{N}$ with $\mathbb{C}^{N_{1}} \otimes \mathbb{C}^{N_{2}}$ under the mixed-decimal representation, which asserts the equalities

$$
|s\rangle_{N}=|k \ell\rangle_{N_{1}, N_{2}}=|k\rangle_{N_{1}} \otimes|\ell\rangle_{N_{2}}
$$

when

$$
s=k N_{2}+\ell, k \in\left\{0, \ldots, N_{1}-1\right\}, \ell \in\left\{0, \ldots, N_{2}-1\right\} .
$$

Remark 2 In the case that $N_{1} \neq N_{2}$, the reader is warned that the operator $\mathcal{F}_{N_{1} \times N_{2}}$ is not equivalent in what follows to $\mathcal{F}_{N_{2} \times N_{1}}$. Specifically, $\mathcal{F}_{N}$ does not commute with the unitary operator $R_{N_{1}, N_{2}}: \mathbb{C}^{N} \rightarrow \mathbb{C}^{N}$ given by

$$
R_{N_{1}, N_{2}}\left|k N_{2}+\ell\right\rangle=\left|\ell N_{1}+k\right\rangle, \quad k \in\left\{0, \ldots, N_{1}-1\right\}, \ell \in\left\{0, \ldots, N_{2}-1\right\},
$$

which interchanges the digits of the mixed-decimal representation.

Notation 3 Let $\mathcal{H}$ be a finite-dimensional Hilbert space. Then $B(\mathcal{H})$ is the Hilbert space of linear transformations on $\mathcal{H}$ with the Hilbert-Schmidt inner product $\langle A, B\rangle_{B(\mathcal{H})}=\operatorname{Tr} A^{\dagger} B .^{4}$

Definition 4 Let $\mathcal{H}$ and $\mathcal{K}$ be finite-dimensional Hilbert spaces, and let $F$ be a nonzero linear transformation on $\mathcal{H} \otimes \mathcal{K}$. An (operator) Schmidt decomposition of $F$ is a decomposition of the form

$$
F=\sum_{k=1}^{\operatorname{Sch}(F)} \lambda_{k} A_{k} \otimes B_{k}, \quad \lambda_{k}>0,
$$

\footnotetext{
${ }^{3}$ This is unitarily equivalent to the discrete Fourier transform.

${ }^{4}$ If $A$ is a linear operator on $\mathcal{H}$, then $A^{\dagger}$ is defined by $\langle x, A y\rangle_{\mathcal{H}}=\left\langle A^{\dagger} x, y\right\rangle_{\mathcal{H}}$ for all $x, y \in \mathcal{H}$. Here $\langle\bullet, \bullet\rangle_{\mathcal{H}}$ is the inner product on $\mathcal{H}$, and we will always take inner products to be linear in the second argument.
} 
where $\left\{A_{k}\right\}_{k=1 \ldots \operatorname{Sch}(F)}$ and $\left\{B_{k}\right\}_{k=1 \ldots \operatorname{Sch}(F)}$ are orthonormal sets ${ }^{5}$ of operators on $\mathcal{H}$ and $\mathcal{K}$ respectively, under the Hilbert-Schmidt inner product. The quantity $\operatorname{Sch}(F)$ is called the Schmidt number, and the $\lambda_{k}$ are called the Schmidt coefficients. Such a decomposition is said to be completely degenerate if $\operatorname{Sch}(F)=(\min (\operatorname{dim} \mathcal{H}, \operatorname{dim} \mathcal{K}))^{2}$ and all the $\lambda_{k}$ are equal. ${ }^{6}$

We remark that the operator-Schmidt decomposition is just a special case of the well-known Schmidt-decomposition

$$
\psi=\sum_{k=1}^{\operatorname{Sch}(\psi)} \lambda_{k} e_{k} \otimes f_{k}, \quad \lambda_{k}>0
$$

of a vector $\psi \in \mathcal{H}_{0} \otimes \mathcal{K}_{0}$, where the $\left\{e_{k}\right\}$ and $\left\{f_{k}\right\}$ are orthonormal. ${ }^{7}$ In particular, one sets $\mathcal{H}_{0}=B(\mathcal{H}), \mathcal{K}_{0}=B(\mathcal{K})$, and $\psi=F \in B(\mathcal{H} \otimes \mathcal{K})$. The decomposition (2) is then obtained by identifying $B(\mathcal{H}) \otimes B(\mathcal{K})$ with $B(\mathcal{H} \otimes \mathcal{K})$ under the natural isomorphism. ${ }^{8}$ It follows that $F$ and $G$ in $B(\mathcal{H} \otimes \mathcal{K}) \simeq$ $B(\mathcal{H}) \otimes B(\mathcal{K})$ have the same operator-Schmidt coefficients, counting multiplicity, iff

$$
A=(\mathbb{U} \otimes \mathbb{V}) B
$$

for some unitary "super-operators" $\mathbb{U} \in B(B(\mathcal{H}))$ and $\mathbb{V} \in B(B(\mathcal{K}))^{9}$

The well-known procedure for computing Schmidt decompositions is reviewed in Theorem 8 of the Appendix. We content ourselves here with the statement that the Schmidt coefficients of $\psi \in \mathcal{H}_{0} \otimes \mathcal{K}_{0}$ are the square roots of the nonzero eigenvalues of the reduced density matrix

$$
\rho_{\psi}=\operatorname{Tr}_{\mathcal{K}_{0}}|\psi\rangle\langle\psi|
$$

Equivalently, the Schmidt coefficients are the nonzero singular values of the operator $B_{\psi}: \mathcal{H}_{0} \rightarrow \mathcal{K}_{0}^{*}$ given by

$$
\left(B_{\psi} f\right)(g)=\langle\psi, f \otimes g\rangle_{\mathcal{H}_{0} \otimes \mathcal{K}_{0}},
$$

\footnotetext{
${ }^{5}$ but not necessarily bases

${ }^{6}$ More generally, if $F: \mathcal{H} \otimes \mathcal{K} \rightarrow \mathcal{H}^{\prime} \otimes \mathcal{K}^{\prime}$, then one may consider decompositions of the form (2), where now the $A_{k}: \mathcal{H} \rightarrow \mathcal{H}^{\prime}$ and $B_{k}: \mathcal{K} \rightarrow \mathcal{K}^{\prime}$ are orthornormal. A useful such decomposition exists for the communication operator $C:\left(\mathbb{C}^{n_{1}} \otimes \mathbb{C}^{n_{2}}\right) \otimes \mathbb{C}^{n_{3}} \rightarrow \mathbb{C}^{n_{1}} \otimes\left(\mathbb{C}^{n_{2}} \otimes \mathbb{C}^{n_{3}}\right)$, defined by $C(a \otimes b) \otimes c=a \otimes(b \otimes c)$. One may check that $C=\sum_{k=1}^{n_{2}} \sqrt{n_{1} n_{3}} A_{k} \otimes B_{k}$, where $A_{k}=n_{1}^{-1 / 2} \sum_{i=1}^{n_{1}}|i\rangle\langle i k|: \mathbb{C}^{n_{1}} \otimes \mathbb{C}^{n_{2}} \rightarrow \mathbb{C}^{n_{1}}$ and $B_{k}=n_{3}^{-1 / 2} \sum_{i=1}^{n_{3}}|k i\rangle\langle i|: \mathbb{C}^{n_{3}} \rightarrow$ $\mathbb{C}^{n_{2}} \otimes \mathbb{C}^{n_{3}}$. Replacing the swap operators in section III.B.3 of 3 by communication operators, one obtains the aforementioned sharp quantum communication complexity bound of 2 .

${ }^{7}$ See 4 for a discussion of the Schmidt decomposition.

${ }^{8}$ In particular, there exists a unique unitary $\Xi: B(\mathcal{H}) \otimes B(\mathcal{K}) \rightarrow B(\mathcal{H} \otimes \mathcal{K})$ such that $(\Xi(A \tilde{\otimes} B))(f \otimes g)=(A f) \otimes(B g)$ for all $f \in \mathcal{H}$ and $g \in \mathcal{K}$. Here $\tilde{\otimes}$ denotes the defining tensor product of $B(\mathcal{H}) \otimes B(\mathcal{K})$, considering $B(\mathcal{H})$ and $B(\mathcal{K})$ as abstract Hilbert spaces.

${ }^{9}$ See exercise 2.80 of 4 . One would like to know much more, i.e. invariants which specify when are there local unitaries $U, Y \in B(\mathcal{H})$ and $V, Z \in B(\mathcal{K})$ such that $A=$ $(U \otimes V) B(Y \otimes Z)$. Such invariants are known only in the two-qubit case, 5 where one has the corresponding canonical decomposition of Khaneja, Brockett, and Glaser 6] (see also Kraus and Cirac [] for simple "magic basis" proof.)
} 
where $\mathcal{K}_{0}^{*}$ is the dual space of continuous linear functionals on $\mathcal{K}_{0} \cdot{ }^{10}$

\section{Schmidt Decomposition of $\mathcal{F}$}

Notation 5 Let $\mathbb{Z}_{N_{1}}=\left\{0, \ldots, N_{1}-1\right\}, \mathbb{Z}_{N_{2}}=\left\{0, \ldots, N_{2}-1\right\}, \mathbb{Z}_{N_{2}}^{2}=\mathbb{Z}_{N_{2}} \times$ $\mathbb{Z}_{N_{2}}, N_{1} \mathbb{Z}^{2}=\left\{\left(N_{1} x, N_{1} y\right) \mid x, y \in \mathbb{Z}\right\},\lceil x\rceil=\min \{n \in \mathbb{Z} \mid n>x\}$, and $\lfloor x\rfloor=$ $-\lceil-x\rceil$. Denote the cardinality of a set $C$ by $|C|$. Its characteristic function $\chi_{C}$ satisfies

$$
\chi_{C}(x)=\left\{\begin{array}{lll}
1 & \text { if } & x \in C \\
0 & \text { if } & x \notin C
\end{array} .\right.
$$

Adopt the convention $n \bmod m \in \mathbb{Z}_{m}$.

Theorem 6 Define an equivalence relation $\sim$ on $\mathbb{Z}_{N_{2}}^{2}$ by

$$
\vec{\ell} \sim \vec{m} \Longleftrightarrow \vec{\ell}-\vec{m} \in N_{1} \mathbb{Z}^{2},
$$

where the subtraction is not modular, and define $\mathcal{M}=\mathbb{Z}_{N_{2}}^{2} / \sim$ to be the set of equivalence classes. ${ }^{11}$ Then a Schmidt decomposition of $\mathcal{F}_{N_{1} \times N_{2}}$ is given by

$$
\mathcal{F}_{N_{1} \times N_{2}}=\sum_{C \in \mathcal{M}} \sqrt{\frac{N_{1}}{N_{2}}|C|} A_{C} \otimes B_{C},
$$

where the matrices of $A_{C}: \mathbb{C}^{N_{1}} \rightarrow \mathbb{C}^{N_{1}}$ and $B_{C}: \mathbb{C}^{N_{2}} \rightarrow \mathbb{C}^{N_{2}}$ are defined by

$$
\begin{array}{ll}
\left(A_{C}\right)_{k_{1} k_{2}}=\frac{1}{N_{1}} \exp \left[\frac{2 \pi i}{N_{1}}\left(N_{2} k_{1} k_{2}+k_{1} \tilde{c}_{2}+k_{2} \tilde{c}_{1}\right)\right] & k_{1}, k_{2} \in \mathbb{Z}_{N_{1}} \\
\left(B_{C}\right)_{\ell_{1} \ell_{2}}=\frac{1}{|C|^{1 / 2}} \exp \left(\frac{2 \pi i}{N} \ell_{1} \ell_{2}\right) \chi_{C}\left(\left(\ell_{1}, \ell_{2}\right)\right) & \ell_{1}, \ell_{2} \in \mathbb{Z}_{N_{2}},
\end{array}
$$

with each $\left(\tilde{c}_{1}, \tilde{c}_{2}\right) \in C$ arbitrarily chosen. ( $A_{C}$ doesn't depend on this choice.)

\footnotetext{
${ }^{10}$ See 4 for a proof that the Schmidt decomposition is a consequence of the singular value decomposition. In fact they are mathematically equivalent.

${ }^{11}$ The reader may check that for $N_{1}=2$ and $N_{2}=3$ that $\mathcal{M}$ consists of $\{(0,0),(0,2),(2,0),(2,2)\},\{(1,0),(1,2)\},\{(0,1),(2,1)\}$, and $\{(1,1)\}$.
} 
Proof. It is trivial to check that $\left\{A_{C}\right\}$ and $\left\{B_{C}\right\}$ are orthonormal sets. Furthermore, for $k_{1}, k_{2} \in \mathbb{Z}_{N_{1}}$ and $\ell_{1}, \ell_{2} \in \mathbb{Z}_{N_{2}}$,

$$
\begin{aligned}
\left\langle k_{1}, \ell_{1}\right| & \left(\sum_{C \in \mathcal{M}} \sqrt{\frac{N_{1}}{N_{2}}|C|} A_{C} \otimes B_{C}\right)\left|k_{2}, \ell_{2}\right\rangle \\
= & \sum_{C \in \mathcal{M}} \sqrt{\frac{N_{1}}{N_{2}}|C|}\left\langle k_{1}\left|A_{C}\right| k_{2}\right\rangle\left\langle\ell_{1}\left|B_{C}\right| \ell_{2}\right\rangle \\
= & \sum_{C \in \mathcal{M}} \frac{1}{\sqrt{N}} \exp \left[\frac{2 \pi i}{N}\left(N_{2}^{2} k_{1} k_{2}+N_{2} k_{1} \tilde{c}_{2}+N_{2} k_{2} \tilde{c}_{1}+\ell_{1} \ell_{2}\right)\right] \chi_{C}\left(\left(\ell_{1}, \ell_{2}\right)\right) \\
= & \sum_{C \in \mathcal{M}} \frac{1}{\sqrt{N}} \exp \left[\frac{2 \pi i}{N}\left(N_{2}^{2} k_{1} k_{2}+N_{2} k_{1} \ell_{2}+N_{2} k_{2} \ell_{1}+\ell_{1} \ell_{2}\right)\right] \chi_{C}\left(\left(\ell_{1}, \ell_{2}\right)\right) \\
= & \frac{1}{\sqrt{N}} \exp \left[\frac{2 \pi i}{N}\left(N_{2} k_{1}+\ell_{1}\right)\left(N_{2} k_{2}+\ell_{2}\right)\right] \\
= & \left\langle k_{1} \ell_{1}\left|\mathcal{F}_{N_{1} \times N_{2}}\right| k_{2}, \ell_{2}\right\rangle,
\end{aligned}
$$

as desired.

The reader may find it instructive to compute the linear spans of the matrices $B_{C}$ corresponding to each of the Schmidt coefficients.

Corollary 7 The Schmidt decompositions of $\mathcal{F}_{N_{1} \times N_{2}}$ fall into three categories:

1. If $N_{1}$ is a factor of $N_{2}$, then there is only one Schmidt coefficient, $\sqrt{N_{2} / N_{1}}$, with multiplicity $N_{1}^{2}$.

2. If $N_{1} \geq N_{2}$, there is only one Schmidt coefficient, $\sqrt{N_{1} / N_{2}}$, with multiplicity $N_{2}^{2}$.

3. Otherwise, $\mathcal{F}_{N_{1} \times N_{2}}$ has three distinct nonzero Schmidt coefficients:

$$
\begin{array}{lll}
\sqrt{\left\lceil\frac{N_{2}}{N_{1}}\right\rceil^{2} \frac{N_{1}}{N_{2}}} & , \quad \text { multiplicity }\left(N_{2} \bmod N_{1}\right)^{2} \\
\sqrt{\left\lceil\frac{N_{2}}{N_{1}}\right\rceil\left\lfloor\frac{N_{2}}{N_{1}}\right\rfloor \frac{N_{1}}{N_{2}}} \quad, \quad \text { multiplicity } 2\left(N_{2} \bmod N_{1}\right)\left(\left(-N_{2}\right) \bmod N_{1}\right) \\
\sqrt{\left\lfloor\frac{N_{2}}{N_{1}}\right\rfloor^{2} \frac{N_{1}}{N_{2}}} \quad, \quad \text { multiplicity }\left(\left(-N_{2}\right) \bmod N_{1}\right)^{2}
\end{array}
$$

In all cases, the Schmidt number of $\mathcal{F}_{N_{1} \times N_{2}}$ is $\min \left(N_{1}^{2}, N_{2}^{2}\right)$. In particular, the Schmidt decomposition is completely degenerate in Cases 1 and 2.

We remark that the previously known cases fall under Case 1 . Case 2 verifies the Schmidt numbers conjectured in 3 . Since the Schmidt decomposition in Case 1 (or Case 2) is completely degenerate, Theorem 8 (below), may be used to find a Schmidt decomposition of the form of equation (2) for any orthonormal basis $\left\{A_{k}\right\}$ (or $\left\{B_{k}\right\}$, in case 2 ). ${ }^{12}$

\footnotetext{
${ }^{12}$ Note that cases 1 and 2 overlap for $N_{1}=N_{2}$.
} 
Acknowledgments: Michael Nielsen's correspondence was greatly appreciated. I would like to thank Mary Beth Ruskai for her comments, which were most useful in making the manuscript more readable. This research was carried out for the Clay Mathematics Institute.

\section{Appendix: A Derivation}

It will soon be apparent that the crucial fact which allows easy calculation of a Schmidt decomposition of $\mathcal{F}$ is the following: No two of the $B_{C}$ have a nonzero matrix entry in the same place.

The well-known computational recipe needed here is summarized in

Theorem 8 Let $\psi \in \mathcal{H} \otimes \mathcal{K}$ be nonzero. If

$$
\rho_{\mathcal{K}} \equiv \operatorname{Tr}_{\mathcal{H}}|\psi\rangle\left\langle\psi\left|=\sum_{\ell \in L} \mu_{\ell}\right| f_{\ell}\right\rangle\left\langle f_{\ell}\right|,
$$

is a spectral decomposition of the reduced density matrix, then a Schmidt decomposition of $\psi$ is given by

$$
\psi=\sum_{\left\{\ell \mid \mu_{\ell}>0\right\}} \sqrt{\mu_{\ell}} e_{\ell} \otimes f_{\ell},
$$

where each $e_{\ell}$ is defined by the requirement that

$$
\left\langle\psi, v \otimes f_{\ell}\right\rangle_{\mathcal{H} \otimes \mathcal{K}}=\sqrt{\mu_{k}}\left\langle e_{\ell}, v\right\rangle_{\mathcal{H}}
$$

for all $v \in \mathcal{H}$. Furthermore, all Schmidt decompositions of $\psi$ may be exhibited in this manner.

Derivation of Theorem 6. We follow the prescription of Theorem 8 and employ the natural isomorphism $B\left(\mathbb{C}^{N_{1}}\right) \otimes B\left(\mathbb{C}^{N_{2}}\right) \simeq B\left(\mathbb{C}^{N_{1}} \otimes \mathbb{C}^{N_{2}}\right)$, as explained in section 1.2. The reduced density superoperator $\rho \in B\left(B\left(\mathbb{C}^{N_{2}}\right)\right)$ is defined by the equation

$$
\langle A, \rho B\rangle_{B\left(\mathbb{C}^{N_{2}}\right)}=\sum_{E}\langle E \otimes A, \mathcal{F}\rangle_{B\left(\mathbb{C}^{N_{1}} \otimes \mathbb{C}^{N_{2}}\right)}\langle\mathcal{F}, E \otimes B\rangle_{B\left(\mathbb{C}^{N_{1}} \otimes \mathbb{C}^{N_{2}}\right),}
$$

for arbitrary $A, B \in B\left(\mathbb{C}^{N_{2}}\right)$, where $E$ runs over a basis of $B\left(\mathbb{C}^{N_{1}}\right)$. For $\vec{j} \in \mathbb{Z}_{N_{1}}^{2}$ and $\vec{\ell} \in \mathbb{Z}_{N_{2}}^{2}$ define the standard basis elements

$$
E_{\vec{j}}=\left|j_{1}\right\rangle\left\langle j_{2}\left|\in B\left(C^{N_{1}}\right), F_{\vec{\ell}}=\right| \ell_{1}\right\rangle\left\langle\ell_{2}\right| \in B\left(\mathbb{C}^{N_{2}}\right) .
$$

We compute $\rho$ by studying its matrix coordinates

$$
\rho_{\vec{\ell} \vec{m}}=\left\langle F_{\vec{\ell}}, \rho F_{\vec{m}}\right\rangle_{B\left(\mathbb{C}^{N_{2}}\right)},
$$


Similarly, let

$$
\mathcal{F}_{\vec{j} \vec{\ell}}=\left\langle E_{\vec{j}} \otimes F_{\vec{\ell}}, \mathcal{F}\right\rangle_{B\left(\mathbb{C}^{N}\right)} .
$$

Then

$$
\begin{aligned}
\rho_{\vec{\ell} \vec{m}} & =\sum_{\vec{j} \in \mathbb{Z}_{N_{1}}^{2}} \mathcal{F}_{\vec{j} \hat{\ell}} \overline{\mathcal{F}}_{\vec{j} \vec{m}} \\
& =\frac{1}{N} \sum_{j_{1}=0}^{N_{1}-1} \sum_{j_{2}=0}^{N_{1}-1}\left(\begin{array}{l}
\exp \left(\frac{2 \pi i}{N}\left(N_{2} j_{1}+\ell_{1}\right)\left(N_{2} j_{2}+\ell_{2}\right)\right) \\
\times \exp \left(-\frac{2 \pi i}{N}\left(N_{2} j_{1}+m_{1}\right)\left(N_{2} j_{2}+m_{2}\right)\right)
\end{array}\right) \\
& =\frac{1}{N} \exp \left(\frac{2 \pi i}{N}\left(\ell_{1} \ell_{2}-m_{1} m_{2}\right)\right) \\
& \times \sum_{j_{1}=0}^{N_{1}-1} \exp \left(\frac{2 \pi i}{N_{1}}\left(\ell_{2}-m_{2}\right) j_{1}\right) \times \sum_{j_{2}=0}^{N_{1}-1} \exp \left(\frac{2 \pi i}{N_{1}}\left(\ell_{1}-m_{1}\right) j_{2}\right)
\end{aligned}
$$

Evaluating the appropriate inverse-Fourier transforms,

$$
\rho_{\vec{\ell} \vec{m}} \equiv \frac{N_{1}}{N_{2}} \exp \left(\frac{2 \pi i}{N}\left(\ell_{1} \ell_{2}-m_{1} m_{2}\right)\right) \times \chi_{N_{1} \mathbb{Z}^{2}}(\vec{\ell}-\vec{m}) .
$$

The spectral decomposition of $\rho$ into a linear combination of projections may be simply read off from the asymptotic $n \rightarrow \infty$ behavior of (6) to the power of $n \in \mathbb{Z}^{+} .{ }^{13}$ One need not do this, however, for using the identity

$$
\chi_{N_{1} \mathbb{Z}^{2}}(\vec{\ell}-\vec{m})=\sum_{C \in \mathcal{M}} \chi_{C}(\vec{\ell}) \chi_{C}(\vec{m})
$$

equation (6) may be rewritten as

$$
\rho=\sum_{C \in \mathcal{M}} \frac{N_{1}}{N_{2}}|C| \times\left|B_{C}\right\rangle\left\langle B_{C}\right|
$$

where the $B_{C}$ are orthonormal, as noted before. The $A_{C}$ are easily computed using (5).

\section{References}

[1] M. A. Nielsen, Ph.D. Thesis, University of New Mexico, 1998, Chapter 6; LANL e-print quant-ph/0011036

[2] M. A. Nielsen, "Entanglement and distributed quantum computation," Talk at the Benasque Center for Physics, July 19, 2000; URL: http://www.qinfo.org/talks/index.html.

\footnotetext{
${ }^{13}$ using matrix multiplication
} 
[3] M. A. Nielsen, C M. Dawson, J L. Dodd, A Gilchrist, D Mortimer, T J. Osborne, M J. Bremmer, A W. Harrow, and A Hines, "Quantum dynamics as a physical resource", (accepted for publication in Phys. Rev. A); LANL e-print quant-ph/0208077v1.

[4] M. A. Nielsen and I. L. Chuang, Quantum Computation and Quantum Information. (Cambridge University Press, Cambridge 2000), pp. 109-111.

[5] Y. Makhlin, LANL e-print quant-ph/0002045 (2000).

[6] N. Khaneja, R. Brockett, and S. J. Glaser, "Time optimal control of spin systems", Phys. Rev. A 63, 032308 (2001); LANL e-print quant-ph quant-ph/0006114

[7] B. Kraus and J. I. Cirac, "Optimal creation of entanglement using a two-qubit gate", Phys. Rev. A 63, 062309 (2001); LANL e-print quant-ph/0011050 